\title{
A spectral view of nonlinear fluxes and stationary-transient interaction in the atmosphere
}

Article

Published Version

Shepherd, T. G. (1987) A spectral view of nonlinear fluxes and stationary-transient interaction in the atmosphere. Journal of the Atmospheric Sciences, 44 (8). pp. 1166-1179. ISSN 15200469 doi: https://doi.org/10.1175/15200469(1987)044<1166:ASVONF>2.0.CO;2 Available at https://centaur.reading.ac.uk/32995/

It is advisable to refer to the publisher's version if you intend to cite from the work. See Guidance on citing.

Published version at: http://dx.doi.org/10.1175/1520-0469(1987)044<1166:ASVONF>2.0.CO;2

To link to this article DOI: http://dx.doi.org/10.1175/1520-

0469(1987)044<1166:ASVONF>2.0.CO;2

Publisher: American Meteorological Society

All outputs in CentAUR are protected by Intellectual Property Rights law, including copyright law. Copyright and IPR is retained by the creators or other copyright holders. Terms and conditions for use of this material are defined in the End User Agreement.

www.reading.ac.uk/centaur 


\section{CentAUR}

Central Archive at the University of Reading

Reading's research outputs online 


\title{
A Spectral View of Nonlinear Fluxes and Stationary-Transient Interaction in the Atmosphere
}

\author{
THEODORE G. SHEPHERD* \\ Center for Meteorology and Physical Oceanography, Massachusetts Institute of Technology, Cambridge, MA 02139
}

(Manuscript received 24 June 1986, in final form 3 November 1986)

\section{ABSTRACT}

Nonlinear spectral transfers of kinetic energy and enstrophy, and stationary-transient interaction, are studied using global FGGE data for January 1979. It is found that the spectral transfers arise primarily from a combination, in roughly equal measure, of pure transient and mixed stationary-transient interactions. The pure transient interactions are associated with a transient eddy field which is approximately locally homogeneous and isotropic, and they appear to be consistently understood within the context of two-dimensional homogeneous turbulence. Theory based on spatial scale separation concepts suggests that the mixed interactions may be understood physically, to a first approximation, as a process of shear-induced spectral transfer of transient enstrophy along lines of constant zonal wavenumber. This essentially conservative enstrophy transfer generally involves highly nonlocal stationary-transient energy conversions.

The observational analysis demonstrates that the shear-induced transient enstrophy transfer is mainly associated with intermediate-scale (zonal wavenumber $m>3$ ) transients and is primarily to smaller (meridional) scales, so that the transient flow acts as a source of stationary energy. In quantitative terms, this transient-eddy rectification corresponds to a forcing timescale in the stationary energy budget which is of the same order of magnitude as most estimates of the damping timescale in simple stationary-wave models (5 to 15 days). Moreover, the nonlinear interactions involved are highly nonlocal and cover a wide range of transient scales of motion.

\section{Introduction}

Synoptic-scale transient disturbances have long been recognized as a crucial ingredient in the circulation of the extratropical troposphere. It is also accepted (e.g. Lorenz, 1967; Hoskins, 1983) that any theoretical framework for understanding the behavior of these features must inevitably come to terms with their strong nonlinearity and apparently random evolution. For this reason, a natural mathematical idealization of the transient synoptic-scale atmospheric flow has been to treat it as a field of two-dimensional homogeneous isotropic turbulence.

The well-known property of two-dimensional turbulence which distinguishes it from three-dimensional (homogeneous) turbulence is the former's inability to cascade kinetic energy to small scales of motion (Taylor, 1917; Lee, 1951), a feature which is due physically to the absence of the vortex stretching mechanism, and which is reflected mathematically in the fact that twodimensional inviscid flow possesses the quadratic invariant of domain-integrated enstrophy $\frac{1}{2}\left(\nabla^{2} \psi\right)^{2}$ as well as that of integrated kinetic energy $\frac{1}{2}|\nabla \psi|^{2}(\psi$ being the streamfunction). These two conservation constraints,

\footnotetext{
- Present affiliation: Department of Applied Mathematics and Theoretical Physics, University of Cambridge, Silver Street, Cambridge, $\mathrm{CB} 3$ 9EW, England.
}

together with a statistical assumption giving an "arrow of time"-such as the broadening of spectral lines (Batchelor, 1953, p. 186), or more generally the increase of a suitable measure of entropy (Carnevale, 1982)imply net nonlinear spectral transfers of energy to larger scales, and of enstrophy to smaller scales (Fjortoft, 1953). These features are indeed qualitatively in accord with atmospheric observations (e.g., Chen and WiinNielsen, 1978; Boer and Shepherd, 1983).

The fact that the large-scale tropospheric flow appears to behave quasi-two-dimensionally is perhaps not too surprising, given the large ratio of horizontal to vertical length scales involved. But the reasons are probably rather more subtle (Charney, 1971) and involve the fact that the extratropical flow tends to be in a state of near geostrophy. Within a framework of stratified quasi-geostrophic turbulence theory (Rhines, 1977, 1979; Salmon, 1980, 1982; Haidvogel and Held, 1980; Herring, 1980; Hoyer and Sadourny, 1982), it can be argued that the (equivalent) barotropic component of atmospheric transient motion may be treated as two-dimensional turbulence forced at the scale of the first internal Rossby radius of deformation-with the forcing arising physically from the process of baroclinic instability.

Other idealized aspects of the two-dimensional theory have been critically studied: effects of Rossby-wave propagation (Rhines, 1975; Holloway and Hendershott, 1977) and of more general anisotropy (Herring, 
1975), of topography (Bretherton and Haidvogel, 1976; Herring, 1977; Rhines, 1977; Holloway, 1978), and of weak horizontal divergence (Holloway, 1983; Farge and Sadourny, 1986), have been introduced in the context of planar geometry. Effects of spherical geometry have also been examined (Tang and Orszag, 1978; Basdevant et al., 1981; Boer, 1983).

All these studies, however, have retained the crucial simplifying assumption of horizontal spatial homogeneity, and it is clear that in the atmospheric context this assumption is highly problematical. Moreover, one can only expect turbulence theory to be relevant-if it is relevant at all-to motions which are "free" in the sense of Lorenz (1979), and which are "mixing" in the dynamical systems sense (e.g., see Salmon, 1982; or in a more general context Prigogine, 1980, pp. 33 ff.), for otherwise the statistical assumptions required to predict cascade directions become untenable. (The assumptions can indeed fail to hold: for example, two-dimensional flow on a beta-plane is provably non-ergodicand therefore non-mixing-for sufficiently small wave steepness (Shepherd, 1987a).) These conditions would suggest that homogeneous turbulence theory should not be applied to quasi-stationary flows forced by topography and heating, for example (perhaps to state the obvious).

Yet the observational studies cited above do lump together all resolved atmospheric motions, irrespective of their dynamical origin and despite their patent inhomogeneity, and still find qualitative agreement with homogeneous turbulence theory. One is naturally led to ask why this is so; this paper attempts to explore precisely that question. It turns out (section 2 ) that the observed spectral transfers are dominated by two dynamically distinct components. The first is that due to nonlinear self-interactions within the transient flow, and the nature of this part of the spectral dynamics does seem to be attributable, in a gross sense, to twodimensional homogeneous turbulence. But the second component-which accounts for about half of the maximum spectral fluxes of kinetic energy and of enstrophy and which indeed dominates the pure transient fluxes at the largest scales of motion-arises from coupled interactions between the stationary and transient parts of the flow, and cannot be sensibly understood within the context of classical (homogeneous) two-dimensional turbulence theory.

Not to put too fine a point on it, one cannot even consider a stationary flow within the context of homogeneous two-dimensional turbulence; such theory makes statements only about the statistical behavior of the flow over timescales longer than the nonlinear mixing timescale (where mixing is used in the dynamical systems sense referred to earlier), for which the time-mean flow must vanish. Homogeneous theory can therefore say nothing about stationary-transient interaction. The reason why an investigation of stationarytransient interaction may be physically meaningful for the atmosphere is that, unlike the (weak) time-mean flow one would obtain from a low-pass filter of twodimensional homogeneous turbulence, the time-mean atmospheric flow does not disappear as the averaging period is lengthened. This is because of the importance of forcing and dissipation, and suggests that the largescale atmospheric flow is essentially "non-mixing".

An important clue for understanding the nature of these mixed stationary-transient interactions comes from the study of Boer and Shepherd (1983; hereafter B\&S), who showed that one may consider the transient flow as being embedded within a larger scale, essentially zonal, stationary flow. It is argued here that this separation in scale, although modest, is nevertheless of great significance, as it allows a rather simple spectral interpretation of the mixed interactions (section 3). Specifically, the interactions may be characterized, to a first approximation, as a shear-induced spectral transfer of transient enstrophy along lines of constant zonal wavenumber, the transfer arising from the shearing of transient vorticity by the stationary flow. In this transfer transient energy is generally not conserved, and there is a net stationary-transient energy conversion.

Indeed, analysis of the spectral observations along the lines suggested by the theory (section 4) provides a determination of the nature and extent of stationarytransient interaction. It is found-at least for the FGGE observing period of January 1979 - that the transients act, in the main, so as to strengthen the stationary flow, and that this "forcing" operates on a timescale comparable to presumed viscous timescales ( 5 to 15 days). Moreover the interactions responsible for this forcing of the stationary flow are highly nonlocal, and they involve a wide range of transient scales. These findings have serious implications for the modeling of low-frequency variability in the atmosphere (section 5).

\section{Observations I: Spectra and nonlinear transfers}

\section{a. Observational data and diagnostic representation}

The global dataset used is essentially that described in B\&S, and covers the special FGGE observing period of January 1979 with samples taken twice daily. The spectra were computed from the NMC FGGE-IIIIa dataset (resolving two-dimensional wavenumbers $n \leqslant 32$ ), while the transfer terms were computed from the more recently available ECMWF FGGE-IIIb dataset (resolving $n \leqslant 40$ ). [It is known that the various FGGE datasets can give quantitatively differing results, especially at high wavenumbers and for sensitive quantities such as the nonlinear transfers (Kung and Tanaka, 1983); but it is only the qualitative features which are of interest here, and these seem to be fairly robust (e.g., Holopainen and Fortelius, 1987).] As in B\&S, kinetic energy and enstrophy spectra and nonlinear transfer terms are produced on each pressure level for each wavenumber $(n, m)$, with $n$ the total spherical harmonic 
index (analogous to $\kappa=\left(k^{2}+l^{2}\right)^{1 / 2}$ in planar geometry), $m$ the zonal wavenumber, and $(n-m)$ the meridional wavenumber. Then the various diagnostics are vertically integrated over the 12 pressure levels from 1000 to $50 \mathrm{mb}$, although this will not be shown explicitly in the formulae below. The resulting diagnostics are not the "barotropic" contributions to the various terms, to be sure, but such a projection is problematical (e.g., see Baer, 1981); the approach adopted here and in B\&S seems to be optimal, if imperfect, although Baer's study suggests (as indeed does Blackmon et al., 1979) that most of the atmospheric kinetic energy does in fact reside in (equivalent) barotropic motion.

The flow may be decomposed into "stationary" and "transient" components, with stationary representing a monthly mean and transient the fluctuations with periods between one day and one month. The latter certainly includes the important band-pass range characteristic of synoptic-scale eddies (e.g., Lau, 1978). On the other hand, low-frequency motions contribute to both components. The energy contribution may then be written as

$$
\begin{aligned}
E(n, m) & =E_{S}(n, m)+E_{T}(n, m) \\
& =\frac{1}{4 a^{2}} n(n+1)\left|\bar{\psi}_{n}^{m}\right|^{2}+\frac{1}{4 a^{2}} n(n+1) \overline{\left|\psi_{n}^{\prime m}\right|^{2}},
\end{aligned}
$$

with the overbar representing the time average, and the prime the deviation therefrom. Here $a$ is the radius of the earth, and $\psi_{n}{ }^{m}$ is the spherical harmonic coeffcient of the streamfunction field for a given pressure level.

Furthermore, the contribution to the energy from meridional and zonal components of motion may be distinguished by defining these respectively (Tang and Orszag, 1978) as

$$
\begin{gathered}
E_{\phi}(n, m)=\frac{1}{8 a^{2}}(2 n+1)|m| \overline{\left|\psi_{n}^{m}\right|^{2}} \\
E_{\lambda}(n, m)=\frac{1}{8 a^{2}}\{2 n(n+1)-(2 n+1)|m|\} \overline{\left|\psi_{n}^{m}\right|^{2}} .
\end{gathered}
$$

It should be noted that these definitions differ from those adopted by Baer (1972). For a discussion of the differences, see Tang and Orszag or B\&S.

If the flow is homogeneous and isotropic, then $\overline{\left|\psi_{n}{ }^{m}\right|^{2}}$ is independent of $m$ and $E(n, m)$ is a function of $n$ alone (Boer, 1983). (Here the time average is deemed to replace an ensemble average.) A simple twopoint measure of the degree of anisotropy is obtained by comparing the meridional and zonal components of energy (2) summed over $m$ at fixed $n$, namely

$$
E_{\phi}(n)=\sum_{m=-n}^{n} E_{\phi}(n, m), \quad E_{\lambda}(n)=\sum_{m=-n}^{n} E_{\lambda}(n, m) .
$$

When $E_{\phi}(n)>E_{\lambda}(n)$ the flow is "meridionally anisotropic", meaning that meridional motions dominate zonal ones at that scale; and when $E_{\phi}(n)<E_{\lambda}(n)$ the flow is instead "zonally anisotropic". Whenever a spectral term is written as a function of a single index alone, summation over the complementary index (as in (3)) is implied. Hence $E(n)=E_{\phi}(n)+E_{\lambda}(n)$ is the energy spectrum associated with total wavenumber $n$.

The contribution to the enstrophy spectrum $G(n$, $m)$ is given simply by $G(n, m)=n(n+1) a^{-2} E(n, m)$, and the above decompositions go through accordingly. All the spectral information is therefore provided by the energy spectrum, unless the spectrum is projected onto anisotropic single indices $m$ or $(n-m)$.

The spectral budget equation for kinetic energy at each wavenumber may be written as

$$
\frac{\partial}{\partial t} E(n, m)=I(n, m)+S(n, m)
$$

where

$$
I(n, m)=\frac{1}{4} \overline{\psi_{n}^{m^{*}}\left\{J\left(\psi, \nabla^{2} \psi\right)\right\}_{n}{ }^{m}}+\text { c.c. }
$$

is the nonlinear interaction term involving other resolved wavenumbers [the braces denoting the spherical harmonic transform and $\mathbf{J}(\cdot, \cdot)$ the two-dimensional horizontal Jacobian operator], and $S(n, m)$ represents all other effects-including interactions with unresolved motions. The enstrophy equation is simply $n(n$ $+1) a^{-2}$ times (4). If the flow is forced and dissipated only over localized parts of the spectrum, then one may hypothesize an equilibrium state where $\partial E(n, m) /$ $\partial t=0=S(n, m)$ over other parts. These latter are then known as "inertial subranges" of the spectrum, and various theoretical approaches are available to treat them (Kraichnan, 1967; Leith, 1968). It has been argued that the atmosphere may have such an inertial subrange connecting forcing and dissipation of enstrophy, but the evidence for this is still inconclusive (see B\&S and refs.; and Shepherd, 1986).

The fact that the interaction terms only redistribute energy and enstrophy among the various resolved wavenumbers is expressed in the fact that

$$
\sum_{n=0}^{N} \sum_{m=-n}^{n} I(n, m)=0=\sum_{n=0}^{N} \sum_{m=-n}^{n} n(n+1) a^{-2} I(n, m),
$$

$N$ being the truncation wavenumber (Platzman, 1960). As a consequence of (6), one may define nonlinear spectral fluxes of energy and enstrophy following projection of the interaction terms onto a one-dimensional representation; for example,

$$
\begin{gathered}
F(n)=-a^{-1} \sum_{\nu=0}^{n} I(\nu)=-a^{-1} \sum_{\nu=0}^{n} \sum_{m=-\nu}^{\nu} I(\nu, m) \\
H(n)=-a^{-3} \sum_{\nu=0}^{n} \dot{\nu}(\nu+1) I(\nu) \\
=-a^{-3} \sum_{\nu=0}^{n} \sum_{m=-\nu}^{\nu} \nu(\nu+1) I(\nu, m)
\end{gathered}
$$


represent, respectively, the fluxes of energy and enstrophy in terms of the two-dimensional index $n$. A positive value of the flux denotes net transfer to smaller scales or to larger $n$, a negative value the reverse. Note that $H(n)$ does not generally equal $n(n+1) a^{-2} F(n)$.

Since $I(n, m)$ is a triple correlation term, its time average can be decomposed into three distinct parts. One may define

$$
\begin{gathered}
I_{S}(n, m)=\frac{1}{4} \bar{\psi}_{n}^{m^{*}}\left\{J\left(\bar{\psi}, \nabla^{2} \bar{\psi}\right)\right\}_{n}{ }^{m}+\text { c.c. }, \\
I_{T}(n, m)=\frac{1}{4} \overline{\psi_{n}^{m^{m}}\left\{J\left(\psi^{\prime}, \nabla^{2} \psi^{\prime}\right)\right\}_{n}{ }^{m}}+\text { c.c. } \\
I_{S T}(n, m)=\frac{1}{4}\left\{\bar{\psi}_{n}^{m^{*}}\left\{\overline{J\left(\psi^{\prime}, \nabla^{2} \psi^{\prime}\right)}\right\}_{n}^{m}\right. \\
\left.+\overline{\psi_{n}^{m^{*}}\left[\left\{J\left(\bar{\psi}, \nabla^{2} \psi^{\prime}\right)\right\}_{n}{ }^{m}+\left\{J\left(\psi^{\prime}, \nabla^{2} \bar{\psi}\right)\right\}_{n}{ }^{m}\right]}\right\}+ \text { c.c., }
\end{gathered}
$$

and it is evident that $I=I_{S}+I_{T}+I_{S T}$. Furthermore, each of these terms is a true interaction term in the sense that (6) holds for it, and the relevant flux functions $F_{S}, H_{S}$, etc. may therefore be defined as in (7).

\section{b. Kinetic energy spectra}

One of the principal findings of B\&S was that the resolved atmospheric motion, when represented in terms of $n$, consists of two quite distinct regimes. For $n<5$, the flow is essentially stationary (which in this context means monthly mean) and appears to be zonally anisotropic, while for $n>8$ the flow is principally transient and is approximately isotropic. The evidence for this is provided by Figs. la, b, which show the decomposition of the energy spectrum $E(n)$ respectively into stationary and transient, and into zonal and meridional, components. (These data were shown also in $B \& S$.) $B \& S$ argued that while the high-wavenumber regime exhibits several of the characteristics of homogeneous isotropic two-dimensional turbulence (including spectral power law behavior not obviously unlike $E(n) \approx n^{-3}$ ), the existence and behavior of the lowwavenumber regime must be explained in other ways. Given the evident scale separation, one might be tempted to assume that the interaction between the stationary and transient components of the flow is small. This turns out not to be the case, however, as will become clear when the nature and extent of the stationary-transient interaction is critically examined in section 4.

Figure 1c shows the zonal-meridional decomposition of the transient energy, and reveals something not recognized in B\&S; namely that the transient energy appears to be approximately isotropic even at the largest scales of motion. This is indeed surprising, because beta-plane turbulence theory (Rhines, 1975; Holloway and Hendershott, 1977) predicts definite zonal anisotropy in the nonturbulent, large-scale regime. Of course the large-scale transients are embedded within the stationary flow and must be affected by it rather strongly; but the naive guess would presumably be that a quasizonal stationary flow would induce zonal anisotropy in the transient flow, in the same sense as beta.

To investigate the observational evidence a little more, Fig. 2 shows the two-dimensional $(n, m)$ spectral representation of the transient energy at the larger scales; this figure is complementary to Fig. 5 of B\&S, which emphasized instead the structure of the total spectrum in the high-wavenumber regime. For isotropy
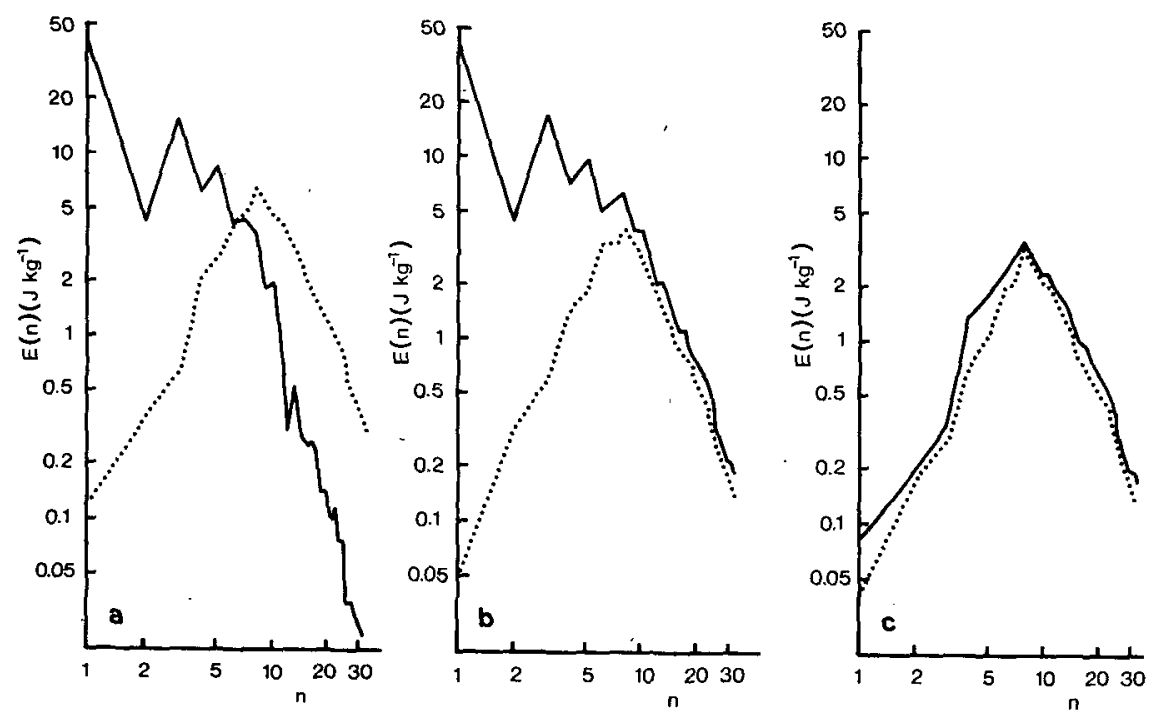

Fig. 1. Spectra of (a) stationary (solid) and transient (dotted) energy, $E_{S}(n)$ and $E_{r}(n)$; (b) zonal (solid) and meridional (dotted) energy, $E_{\lambda}(n)$ and $E_{\phi}(n)$; and (c) zonal (solid) and meridional (dotted) components of transient energy, $E_{T}(n)$ and $E_{T \phi}(n)$. 


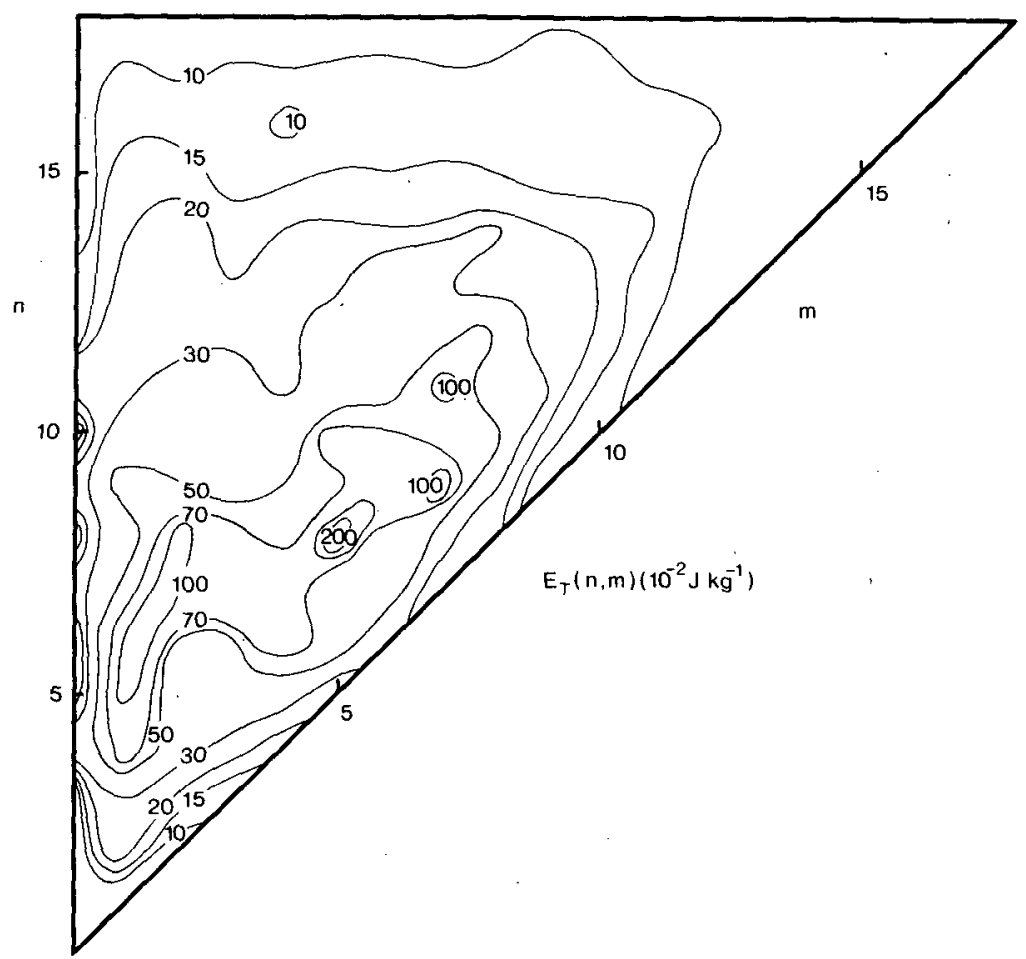

FIG. 2. Two-dimensional spectrum of transient energy, $E_{T}(n, m)$, for the larger scales, in units of $10^{-2} \mathrm{~J} \mathrm{~kg}^{-1}$, quasi-logarithmic contouring. The $m \neq 0$ components. include contributions from positive and negative $m$.

to hold, it is necessary-but not sufficient ${ }^{1}$ - that $E_{T}(n$, $m$ ) be a function of $n$ alone; comparison of $E_{T \phi}(n)$ and $E_{T \lambda}(n)$ only provides a simple two-point estimate. On the basis of Fig. 2 it must be concluded that the transient flow can be truly isotropic only for $n>12$-one may say that these scales are locally isotropic (this is also confirmed by Fig. 5 of B\&S). While all the contours of Fig. 2 tend to run along lines of constant $n$ so that there is approximate isotropy, as suggested by Fig. 1c, there is evidently a considerable amount of anisotropy in the spectrum at the larger scales. The double-peak structure apparent in $E_{T}(n, m)$ around $5 \leqslant n \leqslant 8$ presumably corresponds to the bimodal distribution of transient-eddy energy between low-frequency $(m \approx 2)$ and high-frequency $(m \approx 5)$ components (e.g., Lau, 1978). As will be seen in section 4 (esp. Fig. 8), these two components interact with the stationary flow in quite different ways (cf. also Wallace and Lau, 1985). If the averaging period were extended beyond a month, one would expect the low-frequency energy at small $m$ to increase, but the high-frequency, intermediate- $m$

\footnotetext{
${ }^{1}$ On the sphere, isotropy and homogeneity are equivalent (Boer, 1983). Proof of isotropy therefore requires a demonstration that $\psi_{n}^{m^{*}} \psi_{n^{\prime}} m^{\prime}$ is essentially zero for $(n, m) \neq\left(n^{\prime}, m^{\prime}\right)$. This turns out to be nontrivial, but recent work on the question supports the hypothesis of homogeneity and isotropy at small scales (Boer, 1987).
}

energy characteristic of the band-pass eddies to remain unchanged.

\section{c. Nonlinear fluxes of kinetic energy and enstrophy}

As mentioned in the Introduction, the most striking resemblance between two-dimensional homogeneous turbulence theory and observed atmospheric behavior is the theory's prediction of net up-scale transfers of energy and down-scale transfers of enstrophy. The only relevant scale involved here is $a^{2} / n(n+1)$, as this is the ratio of energy to enstrophy at a given wavenumber. If the turbulence is homogeneous and isotropic then the same picture would emerge when viewed in terms of $m$ (as is traditional in atmospheric spectral energetics: e.g., Saltzman, 1970; Tomatsu, 1979) or $(n-m)$, but this need not be so in general.

The fluxes $F(n)$ and $H(n)$ defined by (7) are shown in Figs. 3a, b, together with their breakdown according to $(8 a-c)$. Given the fact that the greatest part of the transient eddy field is approximately locally isotropic, it is perhaps no surprise that the nonlinear interactions between different transient motions, represented by $F_{T}$ and $H_{T}$, are in the sense predicted by turbulence theory. However there is no reason to expect either of the other pairs of fluxes to behave in this way; and while the pure stationary contributions $F_{S}$ and $H_{S}$ are evidently small, the mixed stationary-transient fluxes $F_{S T}$ and $H_{S T}$ are comparable in strength to the pure transient ones. In- 

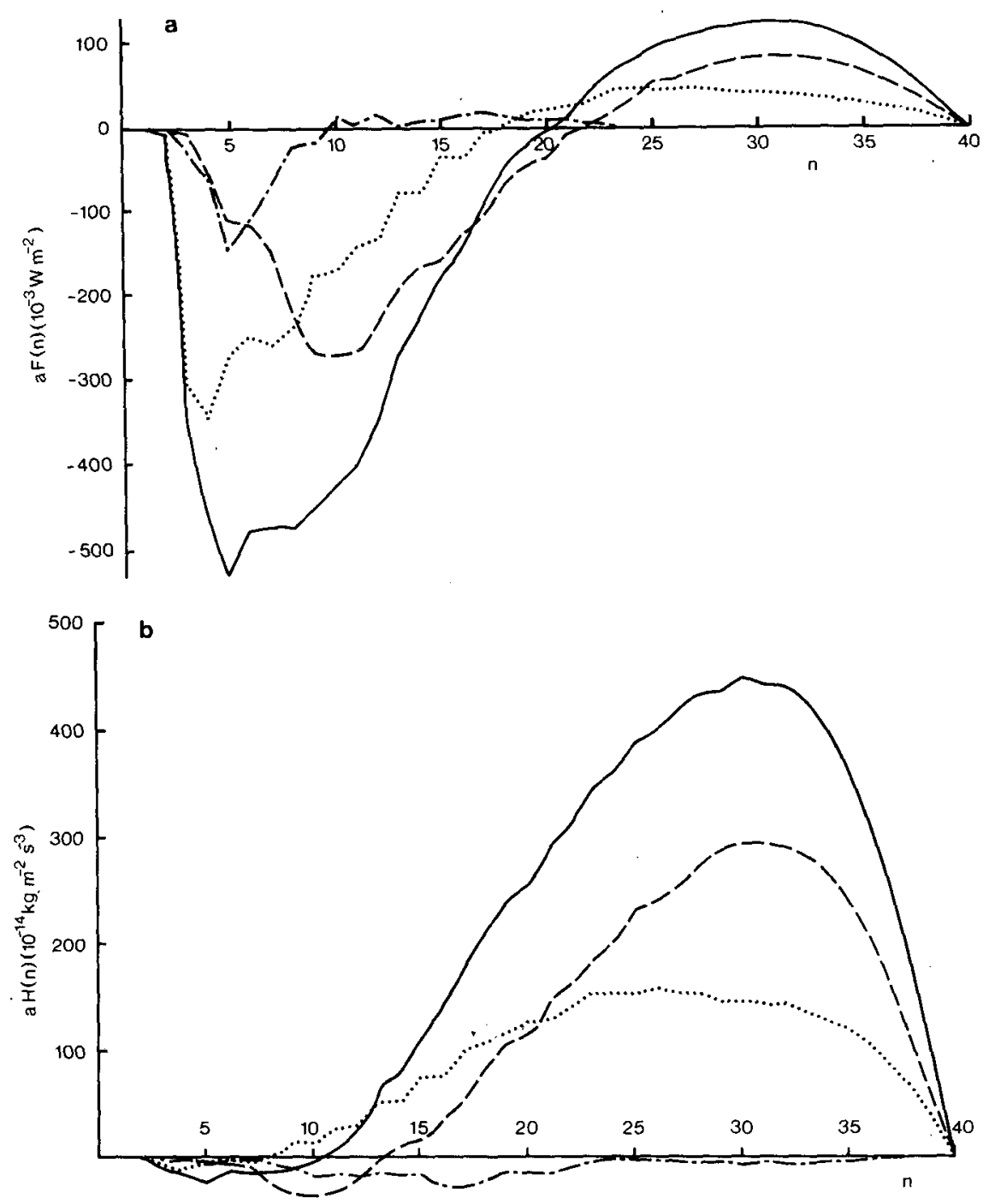

FIG. 3. Nonlinear fluxes (solid) of (a) energy and (b) enstrophy, for a truncation of $N=40$, decomposed respectively into pure stationary (dot-dashed), $F_{S}(n)$ and $H_{S}(n)$, pure transient (dashed), $F_{T}(n)$ and $H_{T}(n)$, and mixed stationary-transient (dotted), $F_{S T}(n)$ and $H_{S T}(n)$, components.

deed, $F_{S T}$ dominates $F_{T}$ at the largest scales, and the apparent penetration of the reverse energy cascade to $n=3$ is seen to be due entirely to the stationary-transient interactions.

\section{A simple picture of stationary-transient interaction for a zonal stationary flow}

The observations of the previous section suggest that one may think of the transient eddy field as evolving in the presence of a larger-scale, principally zonal, stationary flow. It is certainly true that the atmosphere has a strong nonzonal quasi-stationary component, especially in the Northern Hemisphere, and ultimately one would wish to address the problem of its interaction with the transient flow. Indeed an exact theory for finite-amplitude disturbances to nonparallel basic flows now exists (McIntyre and Shepherd, 1987), but its relation to stationary-transient interaction is as yet unclear. The approach followed here is more elementary and proceeds by taking advantage of the fact that most of the stationary energy lies in zonally symmetric ( $m$ $=0$ ) modes, which are of larger scale than the energycontaining transient eddies.

An extensive treatment of planar two-dimensional turbulence in the presence of a large-scale zonal flow has recently been given elsewhere (Shepherd, 1987b; hereafter S87), and the reader is directed there for details of the theory. The principal result is two-fold: first, the stationary-transient interaction may be characterized, to a first approximation, as a shear-induced spectral transfer of transient enstrophy along lines of constant zonal wavenumber $m$, with transient energy generally not conserved; and second, the transient self- 
interactions, provided they are sufficiently turbulent, act in the first instance to spread the transient enstrophy (and energy) along lines of constant total wavenumber $n$. Insofar as the observed transient flow is indeed approximately isotropic for scales smaller than the most energetic ones (Fig. 2), the second part of the result appears to be relevant to the atmosphere. The same applies to the first part, as will be shown in section 4 . But first the general arguments of $\$ 87$ are reviewed briefly here, in order that the result may be understood-for it is in fact exceedingly simple.

The first point to note is that, for transient eddies in the presence of a stationary shear flow, transient energy and enstrophy are not generally conserved by the nonlinear interactions, Integrating over a domain for which boundary terms vanish (such as the surface of a sphere), barotropic theory gives

$$
\begin{gathered}
\frac{d}{d t} \iint \frac{1}{2} \overline{\left|\nabla \psi^{\prime}\right|^{2}} d A=\iint \overline{\psi^{\prime} J\left(\bar{\psi}, \nabla^{2} \psi^{\prime}\right)} d A+S_{E}, \\
\frac{d}{d t} \iint \frac{1}{2} \overline{\left(\nabla^{2} \psi^{\prime}\right)^{2}} d A=-\iint \overline{\nabla^{2} \psi^{\prime} J\left(\psi^{\prime}, \nabla^{2} \bar{\psi}\right)} d A+S_{G},
\end{gathered}
$$

with $S_{E}$ and $S_{G}$ appropriate source-sink terms. The nonlinear source of transient energy in (9a) arises out of shearing of the transient vorticity by the stationary flow, and when positive is the mechanism behind the "Orr effect" of temporary amplification which has been the object of much recent attention in the literature (e.g., Farrell, 1982; Boyd, 1983; Shepherd, 1985).

For a separation in scale between the stationary and transient flow measured by a small parameter $\delta$, scale analysis of the terms in (9) shows that the relative source of transient energy due to the stationary-transient interaction is $\delta^{-2}$ times as large as the relative source of transient enstrophy. Under such conditions, then, the transient enstrophy is approximately conserved (apart from the effects of $S_{G}$, of course). It is interesting to note that this is the opposite situation to that usually considered for weakly-dissipative homogeneous twodimensional turbulence, where one imagines a continual loss of enstrophy due to the down-scale enstrophy cascade to viscous scales while the energy remains approximately constant (e.g., Batchelor, 1969; Bretherton and Haidvogel, 1976).

In planar geometry, nonlinear interactions between different scales of motion of a general flow may be decomposed into wavenumber triads (Lorenz, 1960). In spherical geometry the interactions are no longer quite so simple (cf. Tang and Orszag, 1978), but it is nevertheless true that interactions between a zonal flow $(m=0)$ and a nonzonal disturbance with wavenumber $(n, m)$ may be represented as a sum of triads coupling waves with the same zonal wavenumber $m$. In particular, stationary-transient interactions may be decomposed in this fashion for a zonal stationary flow. Figure 4 shows this decomposition schematically for the case

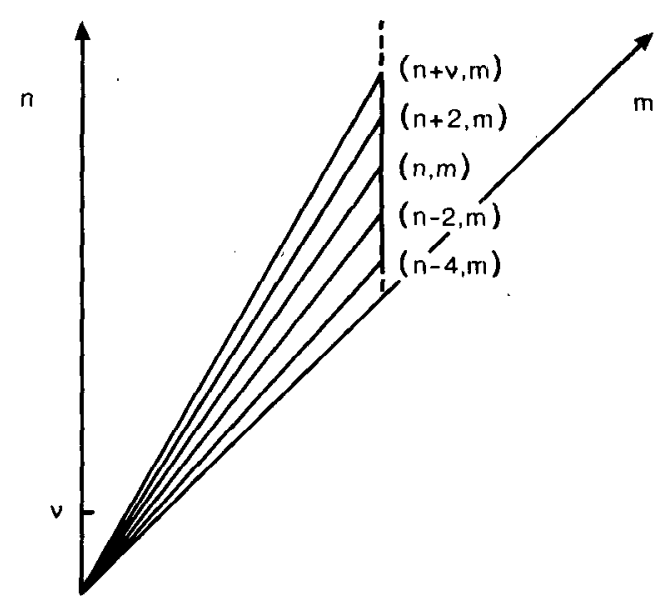

FIG. 4. Possible triad interactions in spherical geometry between a zonal basic flow $(\nu, 0)$ and an arbitrary disturbance component $(n$, $m$ ), represented in terms of positive wavenumbers. Here $v=4$, and so is even, and $n-m$ is presumed to be odd. The basic flow couples disturbances along the vertical line of constant $m$.

of a zonal flow having a single meridional scale $\nu$. In contrast to the planar case, such interactions in spherical geometry may involve nonlocal transient scales, even if $\nu$ is small, for large values of $n-m$.

Now, it was argued above that the transient enstrophy is approximately conserved in the stationarytransient interaction when a scale separation exists between the two flow components. Therefore the simple geometrical constraint depicted in Fig. 4 implies that, in this case, the stationary-transient interaction may be characterized as a shear-induced spectral transfer of transient enstrophy along lines of constant zonal wavenumber $m$. In the case of planar geometry, S87 was able to apply the spectral aspects of ray-tracing theory to deduce the sense of this transfer under certain conditions. The details would be different for spherical geometry, but the basic result is the well-known one that a Rossby wave packet propagating into increasingly westerly zonal flow necessarily has phase lines which lean into the basic shear, and thus increases its wave energy and its meridional wavelength as it propagates, while a packet propagating into increasingly easterly flow will decrease both quantities. This process is the mechanism whereby an initially spectrally-localized Rossby-wave disturbance, under the influence of a zonal shear flow, will spread its enstrophy spectrally along lines of constant zonal wavenumber $m$.

For example, consider a unidirectional shear-induced transfer of transient enstrophy from scale $n_{1}$ to $n_{2}>n_{1}$ taking place along a single zonal wavenumber $m_{1}$. The signature of this process in terms of the diagnostics of section 2 would be approximately as shown in Fig. 5. The enstrophy transfer term $n(n+1) a^{-2} I_{S T}(n$, $m$ ) would be essentially zero for $m \neq m_{1}$; considered as a function of $(n-m)$ for fixed $m=m_{1}$, it would indicate conservative down-scale transfer from scale $n_{1}$ 

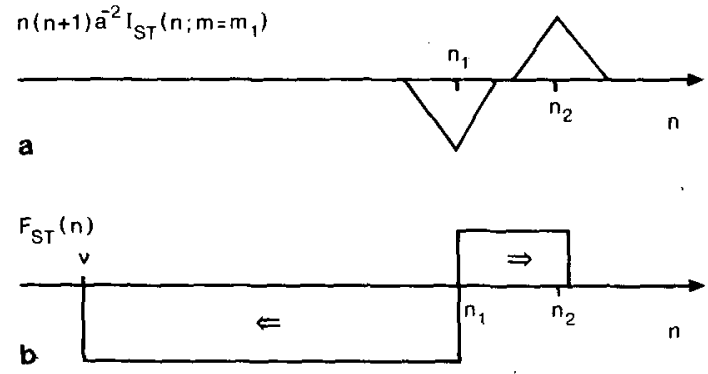

FiG. 5. Schematic of (a) induced down-scale enstrophy transfer, with (b) the associated energy fluxes. Arrows denote the sense of the fluxes.

to $n_{2}$ (Fig. 5a). On the other hand the energy transfer term $I_{S T}(n)$ would have a significant positive component at the scale of the stationary as well as the transient flow, and the energy flux $F_{S T}(n)$ would represent strengthening of the large-scale flow due to straining throughout the induced enstrophy transfer (Fig. 5b).

It is noteworthy that when analysed in terms of $n$ alone, this process would look very much like the cascades of two-dimensional homogeneous turbulence from an initial scale $n_{1}$. The similarity would however be deceptive, as the nonlinear transfer so envisaged is rather different from that obtained by turbulent interactions (although it must of course obey the same overall constraints, which are a property of the governing equations). In the first place, the nonlinear interactions involved are necessarily highly spectrally nonlocal and of a very distinct, anisotropic sort. Second, no statistical assumption analogous to those invoked for turbulent interactions is relevant here, since the mechanism is fundamentally non-mixing; rather, it is systematic, predictable, and dependent on persistence of phase correlations. In particular, for certain parameter regimes (corresponding to an absence of critical lines) ray-tracing theory predicts a reversible vacillation in the sense of the shear-induced spectral transfer.

All this suggests that one may usefully view the nonlinear dynamics of the transient flow as consisting of two distinct, though coupled, elements. The interaction with the stationary flow, to the extent that the latter is large-scale and zonal, may be characterized as a shearinduced transfer of transient enstrophy along lines of constant $m$ associated with highly nonlocal spectral interactions. When this transfer is predominantly to larger $(n-m)$-that is, to smaller scale-then energetic forcing of the stationary flow is implied; when it is to larger scale, then the stationary flow acts instead as a source of transient energy. The second dynamical element is the self-interaction of the transient flow, which may be anticipated-though not assumed-to act in the sense predicted by homogeneous turbulence theory, and which will act in the first instance to spread the transient energy and enstrophy along lines of constant $n$ (and hence across lines of constant $m$ ). As compared with the stationary-transient interaction, this interaction should be relatively local in wavenumber space.

The two dynamical elements will generally interact. For example, the first mechanism by itself inevitably leads to a shear-induced down-scale transient enstrophy cascade and to net energetic forcing of the stationary flow; but S87 demonstrated that the transient self-interactions, if sufficiently turbulent, can force the net stationary-transient interaction to act in quite the opposite sense. Generally the balance appears to be a somewhat sensitive matter (Shepherd, 1984).

\section{Observations II: Stationary-transient interaction}

\section{a. Diagnostic representation}

Before discussing the observations in the light of section 3, the spectral representation of the budgets of stationary and transient energy and enstrophy must be considered. Equation (4) splits naturally into the two parts

$$
\begin{aligned}
& \frac{\partial}{\partial t} E_{S}(n, m)=I_{S}(n, m)+C_{S}(n, m)+S_{S}(n, m), \\
& \frac{\partial}{\partial t} E_{T}(n, m)=I_{T}(n, m)+C_{T}(n, m)+S_{T}(n, m),
\end{aligned}
$$

where the "conversion" terms are given by

$$
C_{S}(n, m)=\frac{1}{4} \bar{\psi}_{n}^{m^{*}}\left\{\overline{J\left(\psi^{\prime}, \nabla^{2} \psi^{\prime}\right)}\right\}_{n}{ }^{m}+\text { c.c., }
$$

$C_{T}(n, m)$

$$
=\frac{1}{4} \overline{\psi_{n}^{m^{*}}\left\{\left\{J\left(\bar{\psi}, \nabla^{2} \psi^{\prime}\right)\right\}_{n}^{m}+\left\{J\left(\psi^{\prime}, \nabla^{2} \bar{\psi}\right)\right\}_{n}^{m}\right\}}+\text { c.c. }
$$

As with (4), the associated enstrophy budgets are obtained simply by multiplying (10) by $n(n+1) a^{-2}$. Here the symbol $I$ denotes an interaction term in the sense that (6) holds for it, so that it leads only to a spectral redistribution of stationary or transient energy and enstrophy; while $C$ denotes conversion in the sense that $\sum C(n)$ and $\sum n(n+1) a^{-2} C(n)$ need not vanish. Obviously there is an essential ambiguity in any such separation, because any part of $I$ itself satisfying (6) can be transferred to $C$, and vice-versa. Note however that $C_{S}+C_{T}=I_{S T}$, so that the stationary and transient budgets may be reconciled at a given wavenumber by writing

$$
\begin{aligned}
\frac{\partial}{\partial t} E_{T}(n, m)=I_{T}(n, m)+ & I_{S T}(n, m) \\
& -C_{S}(n, m)+S_{T}(n, m)
\end{aligned}
$$

in place of $(10 \mathrm{~b})$, if one so desires. (This is essentially the decomposition advocated by Boer, 1987.) But the arguments in favour of this are purely formal. What is important is that whatever formalism one chooses 
highlights the important physical processes in the most clear-cut way. If this be the criterion, then any particular choice can only be preferred on physical grounds for a specific problem.

When there exists a separation in scale between the stationary and transient flows, however, which is indeed the situation here, then a definite physical interpretation of these terms is possible. For in that case, approximate conservation of transient enstrophy implies that $\sum n(n+1) a^{-2} C_{T}(n) \approx 0$ and $\sum n(n+1) a^{-2} C_{S}(n)$ $\approx 0$ separately, so that $n(n+1) a^{-2} I_{S T}(n)$ may be seen as the shear-induced spectral transfer of transient enstrophy described in section 3 . Similarly, insofar as the distributions of $C_{S}(n)$ and $C_{T}(n)$ tend to be correlated with the distributions of $E_{S}(n)$ and $E_{T}(n)$ [a simple consequence of the definitions (11)], and are therefore separated spectrally, $F_{S T}(n)$ represents the stationarytransient energetic exchange associated with straining by highly nonlocal interactions; $F_{S T}(n)<0$ must then imply net energy transfer from the transient flow and is necessarily associated with a net down-scale shearinduced enstrophy transfer (this is the situation depicted in Fig. 5), while $F_{S T}(n)>0$ must imply the opposite.

In this view, $F_{S T}(n)$ should not be interpreted so much as a flux of transient energy (which would be the suggestion from the form (12))-indeed in the case of Fig. 5 the transient component at $n=\nu$, the apparent recipient of such a flux, plays no role whatsoever in the process-but rather as representing the nonlocal stationary-transient energy conversion linking $C_{S}(n)$ and $C_{T}(n)$ at different $n$. On the other hand, in the enstrophy budget $H_{S T}(n)$ can be consistently interpreted as a (local) flux of transient enstrophy, albeit induced by the stationary flow.

\section{b. Stationary-transient interaction and conversion terms}

If the conceptual picture presented in section 3 is at all relevant to the atmosphere, then one should be able to identify in the data the signature of shear-induced spectral transfer of transient enstrophy along lines of constant zonal wavenumber $m$. Previous spectral transfer studies have not been in a position to identify this fundamental process, for a few reasons: first, they have typically not performed the stationary-transient decomposition; and second, the diagnostics have normally been projected onto a single index by summing over the complementary index. Zonal spectral transfers (as functions of $m$ ) will miss the effect entirely, of course, and isotropic ones (as functions of $n$ ) will "smear" the effects of different $m$-lines together. What is needed is rather the examination of $I_{S T}(n, m)$ at fixed $m$, considered as a function of $(n-m)$. Inasmuch as the degree of averaging is much reduced thereby as compared with 1-D transfer representations, one should not expect the diagnostics to be terribly smooth for only one month of data.
Nevertheless the results are remarkably suggestive, despite the evident noise. They are shown in Figs. 6 and 7 for two values of $m, 2$ and 7 , which represent respectively low-frequency and high-frequency transients. Figure 6 shows the energy and enstrophy stationary-transient interaction terms $I_{S T}(n ; m=2)$ and $n(n+1) a^{-2} I_{S T}(n ; m=2)$. The latter term gives only a small average residual when summed over all ( $n$ $-m$ ), demonstrating that it may truly be seen as representing essentially conservative induced transfer of transient enstrophy. This transfer is principally away from the range $10<(n-m)<20$, and is roughly symmetric with respect to $(n-m)$. Figure 6 a verifies that the up-scale transfer is associated with growth of transient energy (the "Orr effect"), and the down-scale transfer with decay; in the net effect, growth dominates decay at $m=2$.

The same diagnostics are shown for $m=7$ in Fig. 7. Again the transient enstrophy is approximately conserved by this process; in contrast to $m=2$, here the transfer is essentially one-way, from $(n-m)<10$ to smaller scales, and there is a significant loss of transient energy to the stationary flow. It would appear that no other outcome was possible, if there was to be any induced transfer at all, because for $m=7$ the principal energy-enstrophy input apparently takes place at the gravest meridional scales.

Figures 6 and 7 characterize the behavior at other $m$. The $m=1,2$ (which are dominated by low-frequency transients) both involve roughly symmetric shear-induced enstrophy transfer and net increase of transient energy; $3<m<12$ (which are dominated by high-frequency transients) are very much like $m$

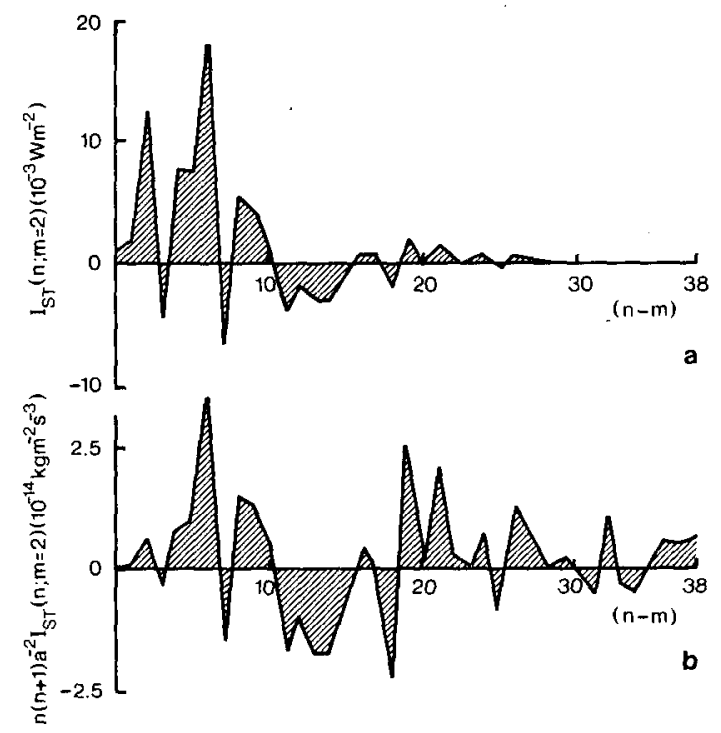

FIG. 6. Stationary-transient interaction terms (a) of energy, $I_{S T}(n$; $m=2)$, and (b) of enstrophy, $n(n+1) a^{-2} I_{S T}(n ; m=2)$, both for fixed $m=2$ as functions of $n-m$. The sum for (b) is 6.8 , so the average enstrophy conversion per mode is 0.18 . 


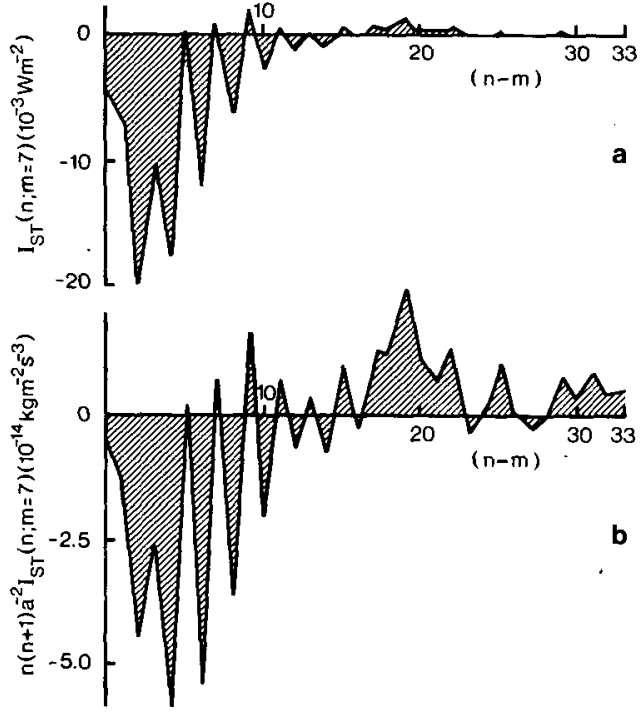

FIG. 7. As in Fig. 6 but for $m=7$. The sum for (b) is -12.0 , the average enstrophy conversion per mode -0.35 .

$=7$, with net down-scale induced enstrophy transfer and corresponding forcing of the stationary flow. The sense of the energy conversion in the two cases is consistent with the results of Wallace and Lau (1985). It may also be noted that the nature of this behavior is qualitatively very similar to that seen in the jet-turbulence simulations of S87. The picture is summarized by the net energy conversion terms $C_{S}(m)$ and $C_{T}(m)$ in Fig. 8, with $C_{T}(m)$ essentially corresponding, at least for $m>1$, to the sum of $I_{S T}(n, m)$ over all $(n-m)$ for a given value of $m$. The fact that $C_{S}(m)$ is dominated by the contribution at $m=0$ is consistent with the fact that the stationary flow consists principally of zonal $(m=0)$ modes. It is seen that there is significant energetic forcing of the stationary flow, which arises from quasi-conservative shear-induced transfer of transient enstrophy to small meridional scales-this transfer taking place mainly in the range $4 \leqslant m \leqslant 8$. For small $m$ there is considerable energy transfer from the stationary flow to large-scale transients. For these data this only reduces the net forcing by a small amount, but for a longer averaging period one would expect an enhancement of the small- $m$ conversion (Wallace and Lau, 1985).

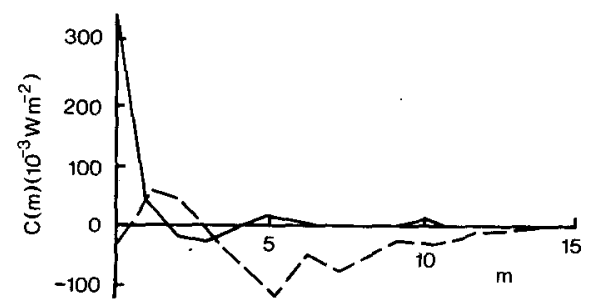

FIG. 8. Energy conversion terms $C_{S}(m)$ (solid) and $C_{T}(m)$ (dashed), as functions of zonal wavenumber $m$, shown only up to $m \leqslant 15$.
It is instructive to consider the energy and enstrophy conversion terms represented as functions of $n$, as in Fig. 9. As expected, the net conversion of enstrophy is fairly small as far as the transient flow is concerned, and the transient enstrophy is primarily redistributed by the stationary-transient interaction. This redistribution is essentially to smaller scales, with the transfer arising principally from two distinct 'source' regions: one around $n=8$, and the other around $n \approx 15$. The first corresponds to the transient energy peak (Fig. 1a), the second roughly to the primary energy input (presumably from baroclinic instability) as inferred from Fig. 3a (strictly from $I(n)$, shown explicitly in Fig. 9a of $\mathrm{B} \& \mathrm{~S})$. It is the transfer from the $n=8$ transient energy peak which is apparently responsible for most of the energetic forcing of the stationary flow (Fig. 9a). It should be pointed out that the enstrophy conversion may well be significant from the perspective of the stationary flow, while being negligible in terms of the transient flow; nevertheless it is convenient to speak of energetic forcing.

Viewed simply in terms of the net stationary-transient interaction, the present results demonstrate that, at least for the January 1979 FGGE observing period, the transients act as a source of kinetic energy (and of enstrophy) for the stationary flow-particularly into the mode $(n, m)=(3,0)$. To estimate the importance of this effect in the overall stationary kinetic energy balance, the following simple calculation is instructive. In the absence of all other effects, the $e$-folding time $\tau$ for the $n=3$ component due to the stationary-transient interaction is given by
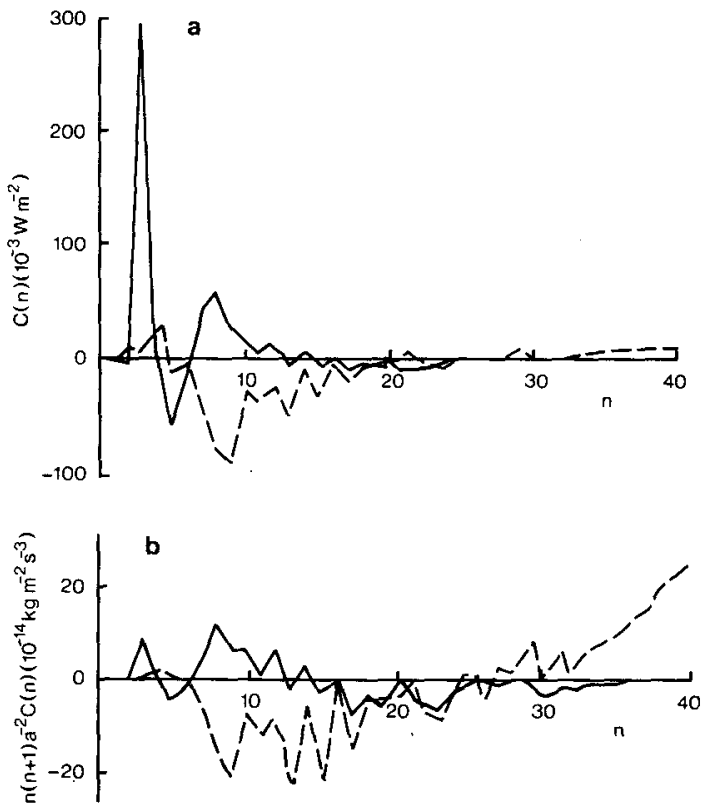

FIG. 9. (a) Energy conversion terms $C_{S}(n)$ (solid) and $C_{T}(n)$ (dashed), and (b) enstrophy conversion terms $n(n+1) a^{-2} C_{S}(n)$ (solid) and $n(n+1) a^{-2} C_{T}(n)$ (dashed), as functions of total wavenumber $n$. 


$$
\tau_{n=3}=\frac{E_{S}(n=3)}{C_{S}(n=3)} \approx \frac{15 \mathrm{~J} \mathrm{~kg}^{-1}}{3 \times 10^{-5} \mathrm{~J} /(\mathrm{kg} \mathrm{s})} \approx 5 \text { days. }
$$

This timescale is of the same order as the spindown timescale normally taken for frictional damping ( 5 to 15 days) in simple models. (If the same estimate is performed for the stationary flow over all scales $n>2-$ $n=1,2$ being apparently uninvolved in this whole process-one gets instead $\tau_{n>2} \approx 15$ days, which is still significant.) While the calculation is only rough, it does suggest that nonlinear interactions with transient eddies should not be neglected in any quantitative theory of the (quasi-) stationary flow which includes frictional effects-as virtually every such theory necessarily does.

Furthermore, Fig. 9a emphasizes that the nonlinear stationary-transient interactions involved in this eddy forcing are spectrally highly nonlocal; this insight indeed merely reflects the physical process which is responsible for the stationary-flow forcing, namely the straining of intermediate-scale transient vorticity by the shear in the stationary flow. It is difficult to reconcile this systematic up-scale energy transfer with the reverse energy cascade of classical two-dimensional homogeneous turbulence, and the superficial resemblance between the two in the atmosphere must be considered fortuitous. (For example, S87 investigated dynamical regimes where the stationary-transient interaction was in the opposite sense to that of the turbulent transient interactions.) Apart from this matter, nonlocalness has another, very practical implication; namely that severely truncated models cannot properly study the (quasi-) stationary flow unless they adequately parameterize the effects of a wide range of transient scales.

\section{Discussion}

This paper began by considering the observed nonlinear spectral transfers of kinetic energy and enstrophy in the atmosphere. The qualitative similarity between the sense of these transfers and those predicted by twodimensional homogeneous turbulence theory is striking, despite the fact that many assumptions required by the theory are not satisfied by the atmospheric flow. It is therefore important to determine the extent to which the observed transfers may reasonably be attributed to the processes encompassed by two-dimensional turbulence, as well as to provide a theoretical basis for understanding the difference.

It has been argued here that two-dimensional homogeneous turbulence theory may be considered to account for the spectral transfers associated with nonlinear interactions between different transient waves, viz. $I_{T}(n, m)$. Interactions between stationary and transient waves-viz. $I_{S T}(n, m)$-cannot be so explained, but are understandable, to a first approximation, as a process of shear-induced spectral transfer of transient enstrophy along lines of constant zonal wavenumber $m$-this transfer involving concomitant stationary- transient conversions of kinetic energy. There is also a component of the spectral transfers due to interactions between different stationary waves, viz. $I_{S}(n, m)$, but it is relatively small and is in any case probably not best studied in a spectral context.

Putting the diagnostics of section 4 together with Fig. 3, a tentative explanation of the observed spectral transfers may be deduced. Transient enstrophy (and energy) is produced at scales around $n \approx 15$ by the process of baroclinic instability. (Recall that, taking a Rossby deformation radius of $1000 \mathrm{~km}$, linear theory predicts most unstable waves of $m \approx 6-8$ in midlatitudes and thus of $n \approx 15$ for isotropic disturbances.) Baroclinic instability theory suggests that these disturbances will tend to be slightly meridionally anisotropic and that they will tend to have phase lines leaning along the horizontal shear (e.g., McIntyre, 1970; Simmons, 1974; Killworth, 1980). Consequently the transient selfinteractions, acting as they will to spread enstrophy along lines of constant $n$ (a process which may be called "turbulent isotropization"), produce enstrophy at small $m$ and $n \approx 15$ with essentially random phase structure. (This process is clearly evident in Fig. 10 of Haidvogel and Held, 1980, for example.) This latter, when acted upon by the shear in the stationary flow, is transferred symmetrically in $(n-\dot{m})$ (Fig. $6 \mathrm{~b})$, and associated with this enstrophy transfer is an amplification of transient energy (Fig. 6a). Since the larger- $m$ waves forced by baroclinic instability will have preferential phase structure however, the shear-induced transfer from those scales will be essentially down-scale, and this indeed dominates over the band $n \approx 15$ (Fig. $9 \mathrm{~b}$ ).

Acting together with the stationary-transient interactions are the transient self-interactions, and indeed the latter are much more 'robust' than the former insofar as rather than relying on persistence of phase structure, they in fact thrive on phase disruption. (See S87 for a fuller discussion.) These interactions appear to be consistently understood in terms of two-dimensional (or, more generally, geostrophic) turbulence theory; not only are the transfers in the correct sense (Fig. 3), but the associated spectra show strong evidence of local homogeneity and isotropy (Figs. 1c, 2). The transient reverse energy cascade is halted rather abruptly around $n \approx 8$, a scale which also--perhaps not surprisingly-corresponds to the spectral peak of transient energy. At this point the phase structure of the waves has presumably been scrambled by nonlinearity, but a significant portion of the spectrum is close enough to the $(n-m)=0$ axis that the shear-induced enstrophy transfers, which pick up when the turbulent transfers weaken, are essentially down-scale (Fig. 7). This is less true for small- $m$ waves, of course, but the net effect of the stationary-transient interactions from these scales is still down-scale enstrophy transfer and associated forcing of the stationary flow (Figs. 8 and 9).

An important question concerns the cause of the transient energy cascade arrest at $n=8$. One is tempted 
to attribute this to the effects of rotation (as described by Rhines, 1975), and indeed there is evidence in Fig. 2 for some accumulation of zonally anisotropic energy around $(n=8, m=2)$. But $\$ 87$ demonstrated that, on the beta-plane, the blocking effects of $\kappa_{\beta}$ are easily overcome by shear-induced transfers associated with a zonal jet-transfers which are indeed evident in these dataand there is no reason to doubt that the same conclusion would apply in spherical geometry. (It should be said however that $\mathbf{S 8 7}$ found the transient self-interactions to be weak for $\kappa<\kappa_{\beta}$, and this feature is consistent with the estimate $n_{\beta} \approx 8$ here.) On the other hand, it was found in Shepherd $(1984,1986)$ that, for a zonal flow consisting of several meridional modes, spectral transfers were arrested at the smallest scale (or largest $n$ ) of the basic flow. This result was purely phenomenological and was not explored in detail, but it seems likely that the same effect may very well play a role here. The subject of the cause behind the transient energy cascade arrest is very much open and demands further investigation.

As for the apparent cascade arrest at $n=3$, seen in the total energy flux (Fig. 3a), it has been shown here to be just a manifestation of the forcing of the $(n, m)$ $=(3,0)$ stationary mode by shear-induced down-scale transfer of transient enstrophy. As such it does not really correspond to a classical homogeneous 'turbulent' reverse energy cascade; indeed the term "cascade" is probably rather inappropriate for this part of the spectral transfer. In fact, one expects the sense of the transfer to $n=3$ to reverse as the averaging period is extended. This is because the interaction with intermediate- $m$ (high-frequency) transients acts so as to strengthen the stationary flow, while the interaction with small- $m$ (low-frequency) transients acts to weaken it. This distinction is consistent with the results of Wallace and Lau. For these data, covering one month, the former interaction is the stronger; but as the averaging period is extended it is expected that the latter will eventually dominate (Wallace and Lau, 1985). In this context, it would be of interest to extend the present study to an entire season, and to isolate the interactions between the stationary, low-pass, and band-pass components of the flow.

The results presented in section 4 demonstrate that the interaction with the transients is strong enough to be quantitatively significant for the stationary flow. This has important implications for the study and modeling of quasi-stationary motions and low-frequency variability, because it suggests that in such studies the effects of a wide range of transient scales of motion must be accounted for-either explicitly, or through a parameterization. It may be noted that a similar conclusion regarding the importance of transients to the stationaryflow balance has been reached from quite independent approaches: by Opsteegh and Vernekar (1982) and by Vallis and Roads (1984) in modeling studies, and by Lau and Holopainen (1984) from an investigation of observed geopotential tendencies; see also Wallace and Lau. An enhancement of the enstrophy cascade and of eddy straining seems also to be linked with northern European blocks (cf. Shutts, 1983).

Acknowledgments. The present research represents part of the author's Ph.D. dissertation, which was completed at the Massachusetts Institute of Technology; he is grateful to his thesis supervisor, Peter Rhines, for much critical advice. The diagnostics were performed with the aid of George Boer and the Canadian Climate Centre, and their continuing hospitality is also much appreciated. Subsequent discussions with Greg Holloway and Mike Wallace have been very helpful in clarifying the interpretation of the results. Funding has been provided at various times by NSF grants ATM76-20070, ATM-81-15712 and OCE-82-19780; by NASA Grant NGR-22-009-727; by NERC Grant GR3/ 5572; and by fellowships from the Natural Sciences and Engineering Research Council of Canada. Finally, the hospitality of D.A.M.T.P. and St. Catharine's College, Cambridge, is gratefully acknowledged during the write-up of this work.

\section{REFERENCES}

Baer, F., 1972: An alternate scale representation of atmospheric energy spectra. J. Atmos. Sci., 29, 649-664.

- 1981: Three-dimensional scaling and structure of atmospheric energetics. J. Atmos. Sci., 38, 53-68.

Basdevant, C., B. Legras, R. Sadourny and M. Béland, 1981: A study of barotropic model flows: Intermittency, waves and predictability. J. Atmos. Sci., 38, 2305-2326.

Batchelor, G. K., 1953: The Theory of Homogeneous Turbulence. Cambridge University Press, $197 \mathrm{pp}$.

- 1969: Computation of the energy spectrum in homogeneous two-dimensional turbulence. Phys. Fluids, 12(Suppl. II), 233239.

Blackmon, M. L., R. A. Madden, J. M. Wallace and D. S. Gutzler, 1979: Geographical variations in the vertical structure of geopotential height fluctuations. J. Atmos. Sci., 36, 2450-2466.

Boer, G. J., 1983: Homogeneous and isotropic turbulence on the sphere. J. Atmos. Sci., 40, 154-163.

_- 1987: Mean and transient spectral energy and enstrophy budgets. Manuscript in preparation.

- and T. G. Shepherd, 1983: Large-scale two-dimensional turbulence in the atmosphere. J. Atmos. Sci., 40, 164-184.

Boyd, J. P., 1983: The continuous spectrum of linear Couette flow with the beta effect. J. Atmos. Sci., 40, 2304-2308.

Bretherton, F. P., and D. B. Haidvogel, 1976: Two-dimensional turbulence above topography. $J$. Fluid Mech., 78, 129-154.

Carnevale, G. F., 1982: Statistical features of the evolution of twodimensional turbulence. J. Fluid Mech., 122, 143-153.

Charney, J. G., 1971: Geostrophic turbulence. J. Atmos. Sci., 28, 1087-1095.

Chen, T.-C., and A. Wiin-Nielsen, 1978: Non-linear cascades of atmospheric energy and enstrophy in a two-dimensional spectral index. Tellus, 30, 313-322.

Farge, M., and R. Sadourny, 1986: Inhibition de la turbulence bidimensionnelle par une rotation d'entrainement. C.R. Acad. Sc. Paris, 302, Sér. II, 847-850.

Farrell, B. F., 1982: The initial growth of disturbances in a baroclinic flow. J. Atmos. Sci., 39, 1663-1686.

Fjortoft, R., 1953: On the changes in the spectral distribution of kinetic energy for twodimensional, nondivergent flow. Tellus, 5, 225-230. 
Haidvogel, D. B., and I. M. Held, 1980: Homogeneous quasi-geostrophic turbulence driven by a uniform temperature gradient. J. Atmos. Sci., 37, 2644-2660.

Herring, J. R., 1975: Theory of two-dimensional anisotropic turbulence. J. Atmos. Sci., 32, 2254-2271.

_ 1977: Two-dimensional topographic turbulence. J. Atmos. Sci., 34, 1731-1750.

,+ 1980: Statistical theory of quasi-geostrophic turbulence. J. Atmos. Sci., 37, 969-977.

Holloway, G., 1978: A spectral theory of nonlinear barotropic motion above irregular topography. J. Phys. Oceanogr., 8, 414-427.

- , 1983: Effects of planetary wave propagation and finite depth on the predictability of atmospheres. J. Atmos. Sci., 40, 314327.

__ and M. C. Hendershott, 1977: Stochastic closure for nonlinear Rossby waves. J. Fluid Mech., 82, 747-765.

Holopainen, E., and C. Fortelius, 1987: On the damping of potential enstrophy in the large-scale transient eddies in the wintertime troposphere. J. Atmos. Sci., submitted.

Hoskins, B. J., 1983: Modelling of the transient eddies and their feedback on the mean flow. Large-Scale Dynamical Processes in the Atmosphere, B. J. Hoskins and R. E. Pearce, Eds., Academic Press, 169-199.

Hoyer, J.-M., and R. Sadourny, 1982: Closure modeling of fully developed baroclinic instability. J. Atmos. Sci., 39, 707-721.

Killworth, P. D., 1980: Barotropic and baroclinic instability in rotating stratified fluids. Dyn. Atmos. Oceàns, 4, 143-184.

Kraichnan, R. H., 1967: Inertial ranges in two-dimensional turbulence. Phys. Fluids, 10, 1417-1423.

Kung, E. C., and H. Tanaka, 1983: Energetics analysis of the global circulation during the special observing periods of FGGE. $J$. Atmos. Sci., 40, 2575-2592.

Lau, N.-C., 1978: On the three-dimensional structure of the observed transient eddy statistics of the Northern Hemisphere wintertime circulation. J. Atmos. Sci., 35, 1900-1923.

- , and E. O. Holopainen, 1984: Transient eddy forcing of the time-mean flow as identified by geopotential tendencies. $J$. Atmos. Sci., 41, 313-328.

Lee, T. D., 1951: Difference between turbulence in a two-dimensional and in a three-dimensional fluid. J. Appl. Phys., 22, 524.

Leith, C. E., 1968: Diffusion approximation for two-dimensional turbulence. Phys. Fluids, 11, 671-673.

Lorenz, E. N., 1960: Maximum simplification of the dynamic equations. Tellus, 12, 243-254.

- 1967: The Nature and Theory of the General Circulation of the Atmosphere. World Meteor. Org., 161 pp.

- , 1979: Forced and free variations of weather and climate. $J$. Atmos. Sci., 36, 1367-1376.

McIntyre, M. E., 1970: On the non-separable baroclinic parallel flow instability problem. J. Fluid Mech., 40, 273-306.

- and T. G. Shepherd, 1987: An exact local conservation theorem for finite-amplitude disturbances to nonparallel shear flows, with remarks on Hamiltonian structure and on Arnol'd's stability theorems. J. Fluid Mech., in the press.

Opsteegh, J. D., and A. D. Vernekar, 1982: A simulation of the January standing wave pattern including the effects of transient eddies. J. Atmos. Sci. 39, 734-744.

Platzman, G. W., 1960: The spectral form of the vorticity equation. J. Meteor., 17, 635-644.

Prigogine, I., 1980: From Being to Becoming. W. H. Freeman, 272 pp.

Rhines, P. B., 1975: Waves and turbulence on a beta-plane. J. Fluid Mech., 69, 417-443.

- 1977: The dynamics of unsteady currents. The Sea, Vol. 6, E. D. Goldberg and co-editors, Wiley, 189-318.

-, 1979: Geostrophic turbulence. Ann. Rev. Fluid Mech., 11, Annual Reviews, 401-441.

Salmon, R., 1980: Baroclinic instability and geostrophic turbulence. Geophys. Astrophys. Fluid Dyn., 12, 167-211.

- 1982: Geostrophic turbulence. Topics in Ocean Physics, A. R. Osborne and P. Malanotte-Rizzoli, Eds., Corso LXXX, Societa Italiana di Fisica, Bologna: North-Holland, pp. 30-78.

Saltzman, B., 1970: Large scale atmospheric energetics in the wavenumber domain. Rev. Geophys. Space Phys., 8, 289-302.

Shepherd, T. G.; 1984: Rossby waves and two-dimensional turbulence in the presence of a large-scale zonal jet. Ph.D. thesis, Massachusetts Institute of Technology, $393 \mathrm{pp}$.

_- 1985: Time development of small disturbances to plane Couette flow. J. Atmos. Sci., 42, 1868-1871.

- 1986: Inhomogeneous two-dimensional turbulence in the atmosphere. To appear in the Proceedings of the 1986 European Turbulence Conference, L. J. Mathieu and G. Comte-Bellot, Eds., Springer-Verlag Proceedings in Physics.

_- 1987a: Non-ergodicity of inviscid two-dimensional flow on a beta-plane and on the surface of a rotating sphere. J. Fluid Mech., in press.

_- $1987 \mathrm{~b}$ : Rossby waves and two-dimensional turbulence in a large-scale zonal jet. $J$. Fluid Mech., to appear.

Shutts, G. J., 1983: The propagation of eddies in diffluent jetstreams: Eddy forcing of "blocking" flow fields. Quart. J. Roy. Meteor. Soc., 109, 737-761.

Simmons, A. J., 1974: The meridional scale of baroclinic waves. $J$. Atmos. Sci., 31, 1515-1525.

Tang, C.-M., and S. A. Orszag, 1978: Two-dimensional turbulence on the surface of a sphere. J. Fluid Mech., 87, 305-319.

Taylor, G. I., 1917: Observations and speculations on the nature of turbulent motion. Scientific Papers, Vol. 2, G. K. Batchelor, Ed., Cambridge University Press, 69-78.

Tomatsu, K., 1979: Spectral energetics of the troposphere and lower stratosphere. Adv. Geophys., 21, 289-405.

Vallis, G. K., and J. O. Roads, 1984: Large-scale stationary and turbulent flow over topography. J. Atmos. Sci., 41, 3255-3271.

Wallace, J. M., and N.-C. Lau, 1985: On the role of barotropic energy conversions in the general circulation. Adv. Geophysics, 28A, 33-74. 